\title{
Application of Cloud Service in Enterprise Informatization
}

\author{
Qiuyue Zheng \\ Harbin University of Commerce College of Marxism Heilongjiang Harbin, China \\ zqy895@163.com
}

\begin{abstract}
Keywords: Enterprise; Informatization; Cloudservice; Application; Strategy
\end{abstract}
\begin{abstract}
With the rapid development of social economy, and the enterprise are more and more focus on the informatization, as well as the demand of information is more and more strong; at the same time, the production and application of cloud services are satisfy the needs of the development of enterprise informatization. In this paper, according to the problems existing in the enterprise informatization construction, as well as the advantages of cloud services and the significance of the use of cloud services, its application in the construction of enterprise-information is discussed and analyzed.
\end{abstract}

\section{Introduction}

Since entering in twenty-first Century, the topic of big data and cloud services will be mentioned again and again. On March 2017, Premier Li Keqiang pointed out in the government work report, to further promote the action"Internet plus" and the national data strategy. Enterprise informatization is the inevitable trend of enterprise development. The cloud services can be required for the enterprise data, resources, etc. are placed on the network, to achieve at any time and place can be data access and computing purposes, so as to promote the development of enterprise-information.

With the development of science and technology and the increasing importance of information technology, most enterprises have entered the stage of effective management and control. For example, due to the wide use of the system, the company must strengthen the construction of information technology in the scientific, normative and effective management and control of security. With the popularity of network applications, information security has also been the focused, the use of firewall, antivirus software, network monitoring, computer hardware and software requirements are higher, with the further improvement of the informatization construction of most companies is becoming more and more mature. However, in this process, we still find that there are still some problems in the process of enterprise-information construction.

\section{Problems in the Enterprise Information Construction}

Lack of Funds. Enterprise informatization is a long-term systematic project, which needs a lot of funds to support its long-term development. If the enterprise in the process of its construction of information reduce its investment due to lack of funds, it will inevitably reduce the efficiency of enterprises, and reduce its economic benefits, so as to bring tremendous pressure on enterprise decision-makers.

Lack of Collaborative Work Management. Many enterprise business systems have their own workflow module, in different professional areas, a number of collaborative applications has been built, but these application systems are two problems, one is the function of repetition, a business often need to log in multiple systems, the other one is unable to exchange, the cross system collaboration cant not be realized.

The enterprise needs is a generic collaborative platform to help professionals to enhance management level, and realize the online messaging and between different majors, in the premise of a unified data base under different departments and collaboration systems.

The Weak of Enterprise Management Foundation. Information is the product of the enterprise business, advanced management ideas and information technology combination, and is closely 
related to the business process, if the business process is not clear or the management mode does not meet the requirements of modern management, it is also difficult to make information. At present, Chinese enterprises are facing such problems in the process of informatization. According to statistics, due to the weak of the basic work of enterprise management, $60 \%$ of domestic enterprise can not get accurate data on time or difficult to obtain data leading to poor operating system or difficult to run. As a result, the first thing to make implement the information success is optimize the process to meet the basic needs of the implementation of information technology to ensure the acquisition of basic data.

The Lack of People-Efficiency of Enterprise Information. On the one hand, due to many enterprises in China have not yet mastered the modern enterprise management methods, the lack of long-term development strategy to take into consideration, so it is very difficult to do the overall planning in promoting the informatization construction, affect the successful implementation of Enterprise informatization construction, created a situation of Enterprise informatization construction of small scale and short time. On the other hand, there is a deviation in the understanding of information technology. Some enterprises still remain in the traditional mode of management and sales, the information construction of enterprises do not pay enough attention; some enterprise leaders eager early in the enterprise-informationization, hold high expectations for information technology, hope to get higher through the benefit of Enterprise informatization, but see investment costs increase, while the benefits did not increase, so as to lose confidence, it is obviously not conducive to the development of informatization construction. All of these leads to serious shortage of investment capital, continued construction of Enterprise informatization will be out of the question.

Far From Reality of Enterprise Information Construction. In the process of informatization construction, many enterprises are not fully aware of the information technology is only a means, and enterprise needs is fundamental, in the process of Enterprise informatization, the system function of foreign goods blindly pursuing big perfection, make the enterprise-information system construction and its business process management, be quite different, so that after the implementation of ineffective, resulting in equipment idle and waste of investment. In the process of promoting Enterprise informatization construction, lack of familiarity with business characteristics, business process, management characteristics of the software company to assist enterprises to promote the process of informatization, information technology to imitate, lack of system demand analysis, the information system construction has no pertinence and practicability, the effect of after-implementation is not ideal.

\section{The Advantages of Cloud Services in Enterprise Information Construction}

\section{Cost Savings}

Cloud services reduce the labor costs of enterprises through virtualization, the cost of buying expensive IT equipment and reduce the cost of enterprise data center applications, which could rapid access to the interests of the company. Cloud services can help companies achieve load balancing on the server, namely enterprise data on the network, do not have to worry about data loss and damage, because the data are generally stored in the "cloud", and "the cloud" is a professional and technical personnel to carry out maintenance and information processing, storage data for the enterprise, their task is to ensure that your data is not destroyed, so the cloud service for the company, is a little money, service good data management apparatus and information service equipment. For example: temporary processing and storage requirements in the holiday season, such as the peak of the processing season, businesses can lease the required resources from the cloud services in order to increase the processing and storage capabilities. This benefit is similar to the application test and the implementation of the block.

In the enterprise-information construction, cloud computing services can solve the problem of energy consumption in the data center by centralized management and centralized use. Through the cloud service, the IT resources are centralized management and sharing, overcome the traditional IT technology, the problem of low efficiency, greatly reduce the energy consumption problem for the 
enterprise. Therefore, the current government computing services also hold positive attitude to the enterprise application cloud, and listed cloud computing in the "12th Five-Year" plan, so we can find that cloud services adapt to the social development which will play a more important role in the information construction in enterprises.

Resource Sharing. Cloud computing services provide a resource sharing function in a flexible and scalable manner.

At present, most enterprises have multiple data centers, one is internal information can not be shared, on the other hand is the data center resources are not scheduling, but through the application of cloud computing services, the resources scheduling between the business systems, extended to realize the resources, so as to improve the efficiency of enterprises can be achieved. At the same time, cloud services also have the ability to load balance, so that resources can be fully utilized.

Cloud services adapt to automated management software, enterprise choose the right software, memory, CPU, etc according to their needs., in the reform or the existing information does not need to be released, and then make a new choice. This allows the business to adjust and reform according to the change of market and business users, cloud computing services to avoid the waste of investment from here, free distribution and expansion can guarantee resource, without having to worry about the loss of data. For example: Virtual Desktop Infrastructure (VDI) is still inconclusive. VDI was originally a "hot" idea to reduce the cost of office software licenses, but later resulted in performance and management issues. However, enterprise CIO is still considering using VDI.

Data Management. Cloud services can provide data management services in the following areas. The first is the information model. The information model is the core of the basic data management, which is the abstract definition of the basic data object, the description of the logical structure and relationship among the objects, and the second is the application of the SkyDrive service. The enterprise uses SkyDrive as the core of the distributed document management data to provide users with high performance document management functions to meet the needs of the management and use of the document data assets. The ledger is a set of professional tools, general ledger management tools, provide account data definition, entry, maintenance and statistical analysis functions can be customized to meet the needs of different professional account management.

Data Fusion and Storage. Now the enterprise is generally developing around the virtually, using the computer to build a huge volume of data center, and on the basis of the virtual data center network technology integration, using the switches, routers and other network infrastructure for cloud services to build data platform to improve customer service experience. The concept of "cloud" is a kind of business model that integrates the resources of IT and provides users with services through distributed processing.

Network is like a binder for "cloud", cloud services provide users with the service technology in the cloud or on the cloud tip. For example: data archiving, assuming that cloud providers can meet the corporate governance standards, some companies are willing to put the historical data from their own data center unloaded to cloud services. The assumption is that these data are not required for large data trend analysis and only for long-term storage purposes.

Although cloud services have such a huge advantage in the process of enterprise information, however, the domestic enterprises in the use of cloud services is still not optimistic. The following is the questionnaire of enterprise cloud service-using, the use of cloud service status is basically showing a triangular shape and distribution of 5:3:2, as shown in Figure 1, there are $8 \%$ enterprises have the use of cloud computing; $10.6 \%$ enterprises are the sum of the two trial, nearly $20 \% .32 .4 \%$ of enterprises in the hesitation hesitate, the proportion of enterprise with no plan reached $49 \%$. According to the survey of cloud service awareness of domestic enterprises, about $45 \%$ of the enterprises do not understand the cloud service or already in use of cloud services without realizing that lead to high proportion of enterprises do not have the cloud service plan. 


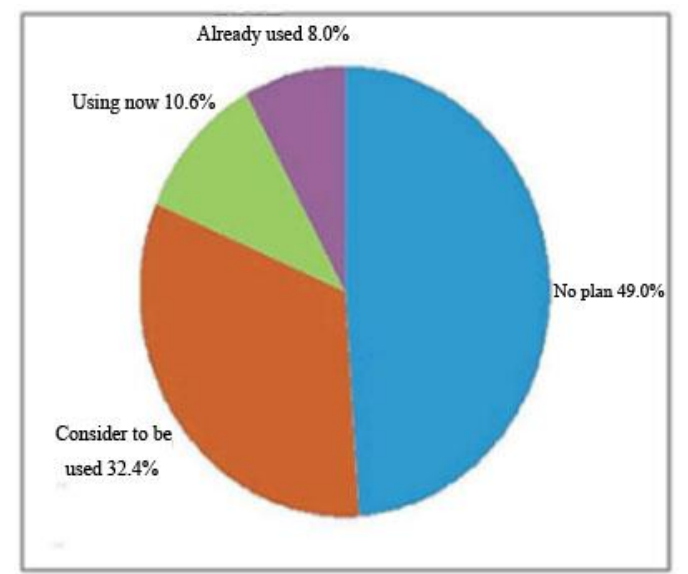

Figure 1. domestic enterprise cloud service usage questionnaire

\section{Measures of Enterprises to Build Cloud Services Platform}

Structured Planning of Cloud Services Platform. Cloud computing is divided into three service modes: SaaS, PaaS, IaaS. SaaS is mainly applied as a service to the customers, IaaS is mainly to the virtual machine resources and provided as a service to users, PaaS is mainly provide developers of application development and deployment platform in the form of services[2]. The cloud service platform should be organized construction, planning and construction should not be blind to words service platform; fully understand these three kinds of service mode, through the use of the construction of the three kinds of service model, meet the application requirements of the cloud services platform, although cloud platform construction is a complicated system engineering, proposed the construction of cloud platforms following the long-term planning and step-by-step implementation of the principle, in accordance with the "elastic cloud" as the input mode.

According to the diverse products, each product may have a variety of models, it is difficult to rely solely on the enterprise-information system of management, so the enterprise can manage the products through the cloud platform, and through the input demand enterprise cloud services platform to realize the original material. The online purchasing; enterprises can also be through the cloud platform products voice, picture, image is introduced to consumers, consumers can understand the product line, consumers can also be input demand cloud services database platform of their own, to enable enterprises to quickly understand the needs of consumers. Companies can link the cloud service platform in the database to the official website of the enterprise data docking, so that consumers are more convenient to search for the relevant information of the required products.

Office Automation to Promote Cloud Services. The template is used to format your paper and style the text. All margins, column widths, line spaces, and text fonts are prescribed; please do not alter them. You may note peculiarities. For example, the head margin in this template measures proportionately more than is customary. This measurement and others are deliberate, using specifications that anticipate your paper as one part of the entire proceedings, and not as an independent document. Please do not revise any of the current designations.

Covering the construction of office automation management system of the whole company, through the daily routine work list into the system in advance, set up a habit of leadership and staff at all levels to use the network to transaction management, make the daily work of the process of scientific and effective analysis and rational planning, through the establishment of the system and in the use process, constantly improve the working process planning, management, improve work efficiency. The enterprise needs to establish perfect personnel management system and reasonable planning of the work content, change the past through the complex, quickly realize the inefficient manual office procedures, all aspects of the acquisition and processing of information, provide the scientific basis for enterprise management and decision-making. For example, the information 
management of Sinopec Tianjin branch of Limited by Share Ltd is mainly through the production process of recording, sorting, statistics and query, and purchasing, materials management, the cash flow information management. Through the production of information management staff will be freed from the complex production management, reducing the operating costs of enterprises, improve work efficiency.

Management of Cloud Resources to Promote the Construction of Cloud Services Platform. In the complex computer architecture, the enterprise must through a powerful management platform to achieve the integration of hardware resources and virtualization of the function of the server's template production deployment. At the same time, there will produce a large amount of data in the process of enterprise operation, and also need to import the data into the cloud platform, using the cloud storage for later collection and management of data, but also analysis the sales of product types according to the data, summed up the consumer tendency of products. According to the results of the analysis, through to the product of the expansion of production scale, to bring greater economic benefits for the enterprise.

Build a Virtual Enterprise, Rational Use of Cloud Services Platform. The formation of the platform construction of virtual enterprise is to multi-industry business services, aims to expand the scope of cooperation enterprise selection in enterprise process in virtual enterprise, the target enterprise choice is more suitable for the needs of the enterprises, reconstruction of virtual enterprise make the enterprise structure to adapt to changing market demand; compared with the traditional enterprise, virtual enterprise has a strong adaptability which can organize a variety of products, the scale of production; and reacted rate in response to environmental changes more agile. The enterprise break the geographical restrictions of enterprises in the construction process through the service platform, increase the number of choices in cooperative enterprises, enterpriseinformation system based on Virtual Enterprises with core competitiveness as the goal, to build cloud service platform for virtual enterprise. The virtual enterprise can be developed based on information network, enterprise operation through information sharing, data exchange data using the general information achievements of the enterprise, convenient to communicate with the enterprise production and marketing business, to achieve unity through cooperation between various enterprises, cooperative enterprises to ensure harmonious development, enhance the whole competition ability of an enterprise.

Before entering the cloud service platform, we should in accordance with the cloud service data standards for cloud services resources, so that the input resources in line with the cloud service platform application standards. When the cloud service platform is done, enterprises will analyze the needs of the enterprise, to find solutions through virtual communication between business solutions to become very easy, when the data exchange, cloud services platform middleware related tasks, to ensure the safety and accuracy of cloud services.

In fact, in addition to the above information which, in order to promote the development of Enterprise informatization through the construction of the cloud service measures, the leaders should have a deep understanding of the Enterprise informatization essence, inevitability and necessity to fully understand the enterprise-information; to continue to strengthen the understanding of the enterprise employees through various measures, will unify up informatization construction. enterprise-information is a full range of innovation and change, first of all, the lead in decisionmaking need to deepen the sense of information, and then to promote the participation of employees, so that enterprise-information can be successfully carried out and made achievement.

\section{Conclusion}

The application and promotion of cloud services has brought a revolutionary effect to the Enterprise informatization, and the upfront costs of cloud services is very low, easy to upgrade, as well as solve a lot of enterprises IT tight budget problems, so the cloud service in the enterprise-information construction plays a very important role. Through the construction of enterprise cloud services, the management of production will be more automated, informational, so as to achieve greater 
economic benefits. In the future, the scope of cloud services will be more and more widely used in enterprises, the form will be more diverse, the content will be richer.

\section{References}

[1] Zhang Jian. Cloud Computing, Analysis of Thinking and Influence, [J]. Telecom Network Technology, 2012 (18)15-18.

[2] Li Qiao, Zheng Xiao. Summary of Current Research on Cloud Computing [J]. Computer Science, 2011 (14)32-37.

[3] Zhao Jie, Yu Haitiao. Enterprise Architecture: enterprise information, strategic planning, governance and overall architecture design of information system. [M]. Beijing: Publishing House of electronics industry, 2012

[4] Wei Ling, Hui Shihai. The Problems of Implementing ERP in China's Small and Medium Enterprises and Solutions. [J] Technology and Management, 2013 (90)45-46.

[5] Song Lizhi, The Application of Cloud Computing in the Financial Industry [J]. Financial Technology Times, 2011, (11)60-61.

[6] Ma Xiujuan, Enterprise Information. Based on Cloud Computing [J]. Electromechanical Archives, 2013 (1)58-60.

[7] Liang Jiewen, Tong Detian, Review of Information Security under the Cloud Computing [J]. Information and Computer: theory edition, 2012 (12)18-19.

[8] Chen Jing, The ERP System to Promotes the Small and Medium-sized Enterprise Informatization Construction and Optimization [J]. Chinese Management Information, 2013 (7) 90

[9] Li Qiao, Zheng Xiao, Summary of Current Research on Cloud Computing [J]. Computer Science, 2011.38 (4)32-37.

[10] Hu Lei. Thinking and Exploration of Informatization of Small and Medium Enterprises in China [J]. Business Economics, 2011 (11)27-29. 